\title{
Hierarchical clustering in the Local Group dwarf galaxy NGC 6822
}

\author{
S. Schmeja, ${ }^{1}$ D. A. Gouliermis,${ }^{2}$ R. S. Klessen, ${ }^{1}$ W. J. G. de Blok ${ }^{3}$ \\ and F. Walter ${ }^{2}$ \\ ${ }^{1}$ Zentrum für Astronomie der Universität Heidelberg, Heidelberg, Germany \\ ${ }^{2}$ Max-Planck-Institut für Astronomie, Heidelberg, Heidelberg, Germany \\ ${ }^{3}$ Department of Astronomy, University of Cape Town, Cape Town, South Africa
}

\begin{abstract}
Star formation appears to be clumped into a hierarchy of structures, from giant molecular clouds down to individual cores and clusters, which are often hierarchical themselves, showing significant substructure. This has been demonstrated for our Galaxy through the application of sophisticated statistical methods, in particular the nearest-neighbour density and the minimum spanning tree (MST), to different star-forming regions. Here we present our analysis of clustered star formation as demonstrated through the detection of structures of young stellar populations in the dwarf star-forming galaxy NGC 6822 .
\end{abstract}

Keywords. galaxies: Local Group, galaxies: star clusters, galaxies: stellar content, galaxies: structure

The full poster (in pdf format) is available at http://www . astro.iag.usp.br/ iaus266/Posters/pSchmeja.pdf. 